A phase II baseline versus treatment study to determine the efficacy of Raltegravir (Isentress) in preventing progression of relapsing remitting multiple sclerosis as determined by gadolinium-enhanced MRI: The INSPIRE Study

Julian Gold, Monica Marta, Ute C. Meier, Tove Christensen, David Miller, Daniel Altmann, David Holden, Lucia Bianchi , Rocco Adiutori, David MacManus, Tarek Yousry, Klaus Schmierer, Benjamin Turner, Gavin Giovannoni

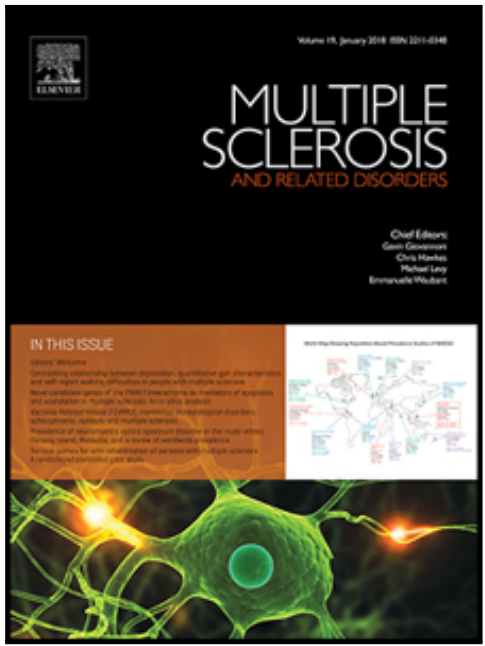

PII:

DOI:

Reference:

To appear in:

Received date:

Revised date:

Accepted date:
S2211-0348(18)30179-2

10.1016/j.msard.2018.06.002

MSARD 857

Please cite this article as: Julian Gold, Monica Marta, Ute C. Meier, Tove Christensen, David Miller, Daniel Altmann, David Holden, Lucia Bianchi, Rocco Adiutori, David MacManus, Tarek Yousry, Klaus Schmierer, Benjamin Turner, Gavin Giovannoni, A phase II baseline versus treatment study to determine the efficacy of Raltegravir (Isentress) in preventing progression of relapsing remitting multiple sclerosis as determined by gadolinium-enhanced MRI: The INSPIRE Study , Multiple Sclerosis and Related Disorders (2018), doi: 10.1016/j.msard.2018.06.002

This is a PDF file of an unedited manuscript that has been accepted for publication. As a service to our customers we are providing this early version of the manuscript. The manuscript will undergo copyediting, typesetting, and review of the resulting proof before it is published in its final form. Please note that during the production process errors may be discovered which could affect the content, and all legal disclaimers that apply to the journal pertain. 


\section{A phase II baseline versus treatment study to determine the efficacy of Raltegravir (Isentress) in preventing progression of relapsing remitting multiple sclerosis as determined by gadolinium-enhanced MRI:}

\section{The INSPIRE Study}

Julian Gold ${ }^{1,5}$, Monica Marta ${ }^{1}$, Ute C. Meier ${ }^{1}$, Tove Christensen ${ }^{4}$, David Miller ${ }^{2}$, Daniel Altmann ${ }^{3}$, David Holden $^{1}$, Lucia Bianchi ${ }^{1}$, Rocco Adiutori ${ }^{1}$, David MacManus ${ }^{2}$, Tarek Yousry ${ }^{2}$, Klaus Schmierer ${ }^{1}$, Benjamin Turner ${ }^{1}$, Gavin Giovannoni ${ }^{1}$

Institute(s): ${ }^{1}$ Neuroscience and Trauma, Queen Mary University of London, Blizard Institute, Barts and the London School of Medicine and Dentistry, ${ }^{2}$ Institute of Neurology. University College London, ${ }^{3}$ Medical Statistics Department, London School of Hygiene and Tropical Medicine, London, United Kingdom, ${ }^{4}$ Department of Biomedicine, Aarhus University, Aarhus, Denmark, ${ }^{5}$ The Albion Centre, Prince of Wales Hospital, Sydney, NSW, Australia.

Abstract

Background: Although the aetiology of Multiple Sclerosis (MS) remains elusive, it is clear that Epstein Barr Virus (EBV) and possibly other viruses play a role in the pathogenesis of MS. Laboratory evidence suggests that Human Endogenous Retroviruses (HERVs) could also have a role, but no interventional therapy has determined what will happen if HERVs are suppressed. Recent epidemiological evidence indicates patients with HIV infection have a significantly lower risk of developing MS and that HIV antiretroviral therapies may be coincidentally inhibiting HERVs, or other retroelements, that could be implicated in MS.

Objectives: To systematically investigate the effects of an HIV integrase strand inhibitor, raltegravir, on the number of gadolinium (Gd)-enhanced MRI lesions in people with active relapsing MS.

Methods: This is a Phase 2a clinical trial where twenty participants were enrolled in a 3 month baseline phase followed by 3 months of treatment with raltegravir $400 \mathrm{mg}$ twice a day. Patients had monthly Gd-enhanced MRI, saliva collection to test for EBV shedding, blood sampling for safety monitoring, virology (including HERVs), measurement of immunological and inflammatory markers; and physical, neurological and quality-of-life assessments.

Results: All patients completed the six months trial period.

The primary outcome measure of MS disease activity was the number of Gd-enhancing lesions observed, and raltegravir had no significant effect on the rate of development of Gd-enhancing lesions during the treatment phase compared with the baseline phase.

Additionally, there was no change in secondary outcomes of either disability or quality-of-life measures that could reasonably be attributed to the intervention. There was a significant positive between HERV-W/MSRV (Multiple Sclerosis Related Virus) Gag Flix (Fluorescence index) B cells and the number of $G d$-enhanced lesions at any visit $(p=0.029)$, which was independent of any potential influence of the trial drug administration. Regarding EBV shedding, there was no significant correlation between the amount of EBV shedding and the number of lesions. No change was detected in inflammatory markers (IL-8, IL-1 $\beta$, IL-6, IL-10, TNF, IL-12p70 and HCRP), which were all within normal limits both before and after the intervention. Serum CD163 expression was also unchanged by raltegravir.

Conclusions: Raltegravir did not have any impact on MS disease activity. This could be due to the choice of antiretroviral agent used in this study, the need for a combination of agents, as used in 
treating HIV infection, the short treatment period or dosing regimen, or the lack of a role of HERV expression in MS once the disease is established. Borderline significance for the association between EBV shedding and the total number of lesions, probably driven by new lesion development, may indicate EBV shedding as a marker of inflammatory disease activity. In conclusion, interesting correlations between HERV-W markers, EBV shedding and new MRI lesions, independent from treatment effects, were found.

\section{Background}

2018 will be the $150^{\text {th }}$ anniversary since Jean-Martin Charcot (1825-1893) published the triad of signs which denote multiple sclerosis (1); although Alastair Compston observes the actual timeline for describing MS cannot be so clearly attributed to Charcot (2). What is indisputable, however, is that in the past 150 or more years we have moved only marginally closer to finding the aetiology of MS. Moreover, there remains considerable debate about the pathogenesis of the disease; to the extent of no broad consensus on whether the different manifestations of MS are iterations of a single disease entity or, in fact different diseases, with the only consensus being that MS is a complex interaction between a genetic predisposition and environmental factors (3) resulting in an autoimmune dysregulation targeting myelin. One of the more prevailing theories about the aetiology of MS is an as yet unconfirmed virus that triggers or causes the disease cascade (4). The most likely viral candidate is a herpes virus, possibly Epstein-Barr virus (EBV) which infects human primary B-cells and maintains a lifelong and persistent infection in memory B cells (5), depletion of memory B-cells seem to be increasingly associated with the pathogenesis of MS (6). However, the causal link between EBV and MS is unclear and until there is either an effective treatment or vaccine to control EBV, the nature of any association will remain unresolved.

An inter-related theory about the aetiology of MS involves the role of human endogenous retroviruses (HERVS) which may be transactivated by EBV and triggered to express HERV encoded proteins. These proteins are considered as 'foreign' by the immune system and may trigger a B-cell response. The resulting antibodies may then cross-react with other proteins, inducing the autoimmune response that results in destruction of myelin. This process is called 'molecular mimicry' (7). Additionally, the HERV encoded proteins may act as superantigens (8, 9), and HERV encoded proteins may have neuropathogenic potential (10). Initial evidence for the possible involvement of HERVs in MS was suggested by Perron et al. in $1991(11,12)$ and by Christensen et al. $(13,14)$, but there has been no conclusive evidence. A recent meta-analysis on studies of the role of HERVs in MS concluded that adequate data were available to show there is an association between expression of HERVs, notably the HERV-W family and MS (15). The HERV-W family comprise a number of sequences of HERV-W/MSRV/Syncytin-1, originating from a mosaic of loci, essentially indistinguishable from a single locus by the methods hitherto used (16). The two HERV families HERV-W/MSRV and HERV-H/F are activated in MS, and levels of HERV antigens are increased on the peripheral blood mononuclear cells (PBMCs), particularly on B cells and monocytes $(17,18)$.

This temporal cascade of laboratory evidence has been paralleled by clinical observations that describe MS-like presentations in patients who were diagnosed with the exogenous retrovirus Human Immunodeficiency Virus (HIV) infection (19). In these patients, it was presumed the MS symptoms were most likely manifestations of HIV-encephalopathy. In 2012, a case report of a man who was confirmed to have both HIV and RRMS and appeared to go into long term remission from his RRMS when he was treated with HIV antiretroviral (ARV) therapy (20). The hypothesis is that ARVs may inhibit HERVs as well as inhibiting HIV and thus ARVs may ameliorate RRMS. In order to explore this observation further a Phase 2 baseline versus treatment clinical trial was planned to 
investigate the role of the Integrase inhibitor, raltegravir, in patients with active RRMS, as defined by the presence of a gadolinium enhanced lesion on MRI scanning at baseline.

Raltegravir (Isentress ${ }^{\circledR}$, Merck and Co., Inc., Whitehouse Station, NJ, USA) is a twice daily oral integrase strand transfer inhibitor approved for treating HIV infection in 2007, in combination with other antiretroviral agents. Its safety profile has been confirmed during more than 10 years of use (21). Raltegravir was chosen as it has a proven safety profile and, as there are not available in-vitro data on the effectiveness of antiretroviral therapy in HERVs, we decided that a single agent would be appropriate for an initial investigation as was also the case in the initial clinical studies of HIV.

\section{Participants and Methods}

Primary efficacy outcome: To determine a reduction in the number of new or recurrent Gdenhancing lesions that appear on brain T1-weighted MRI over the period of treatment with raltegravir, compared to baseline.

Secondary efficacy outcomes: Determine the cumulative number of new or enlarging $\mathrm{T} 2$ weighted lesions on brain MRI. Determine specific inflammatory markers of MS activity. Determine specific indicators of endogenous retroviral activity and activity of herpes viruses. Determine Multiple Sclerosis Functional Composite (MSFC), Kurtzke Extended Disability Status Scale (EDSS), Multiple Sclerosis Quality of Life Inventory (MSQLI).Participants

Adult patients 18-55 years of age with active RRMS according to the revised McDonald Criteria (2010), determined as having at least one documented relapse within the last 12 months or at least one Gd-enhancing lesion on brain MRI during the 3 months prior to screening and an EDSS score not exceeding 6.0, were eligible to enrol in the study. Additionally, participants had to meet standard inclusion and exclusion criteria. After enrolment in the study, participants had a baseline Gdenhanced MRI and if there was an active lesion they entered a 3 months observation period, during which they received no raltegravir, followed by 3 months on treatment. All participants were recruited at the Clinical Research Centre of the Royal London Hospital and were drawn from the catchment area of greater London. A total of 31 participants were screened, of these 8 had no evidence of $\mathrm{Gd}$ enhancing lesions in their baseline MRI and were screen failed. Of the 23 participants who were recruited into the study 3 were withdrawn prior to starting the treatment phase; one at the request of the participant and the remaining two due to MS relapse. Medications for spasticity, bladder control, fatigue and other MS symptoms were permitted. Administration of oral or IV steroids for clinical exacerbation of MS was also permitted as follows: for oral administration. up to $500 \mathrm{mg}$ of methylprednisolone daily for no more than 5 days; for IV administration: up to $1 \mathrm{~g}$ of methylprednisolone daily for no more than 3 days.

This study was given ethical approval by NRES Committee East of England - Cambridge East. Further amendments were made which also gained ethical approval from the same Ethics Committee.

Clinical Assessment

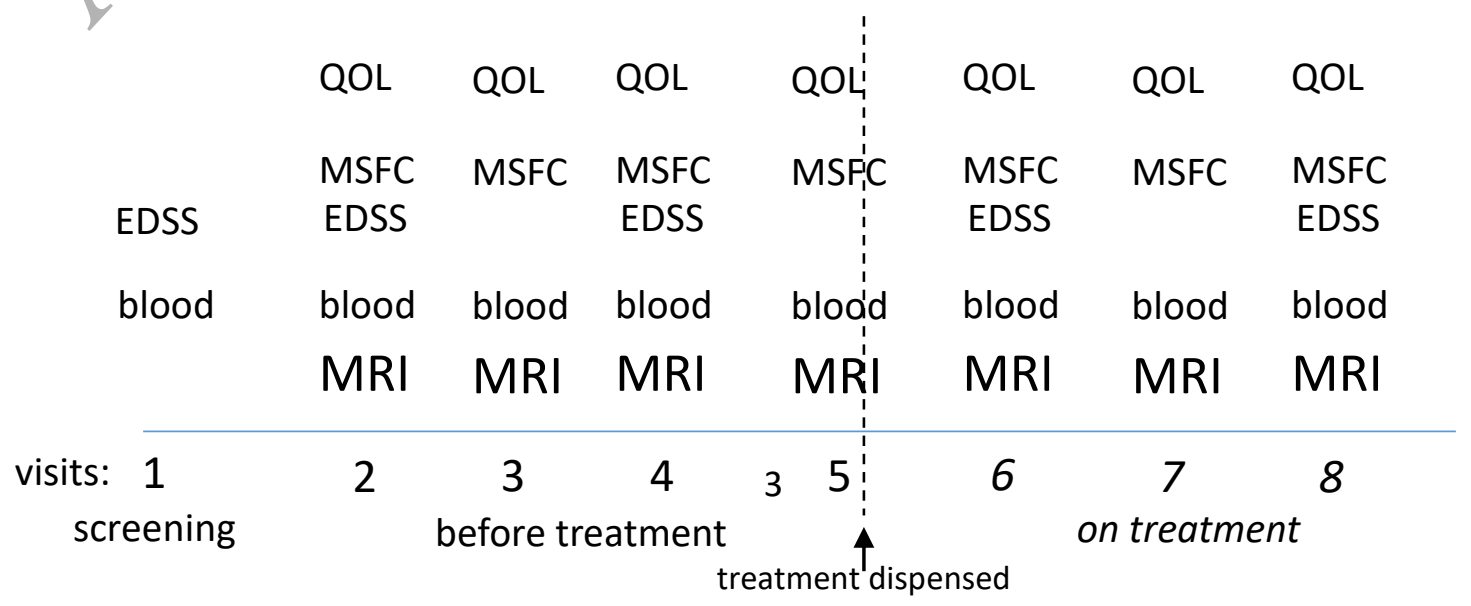


During the pretreatment observation period (day 0 to day 84 ) participants attended 3 visits at baseline, day 28 and day 56. Treatment was first dispensed at the fourth visit, on day 84 , after which patients were treated with open label raltegravir $400 \mathrm{mg}$ twice daily; participants then attended three visits during the treatment period, at day 112, 140 and 168 (end of study).Gd enhanced brain MRIs were performed every 4 weeks from baseline through study day 168 (end of study visit). Only MRIs of the brain were performed. MRI performed during the pretreatment observation period provided data on the baseline number of lesions and the rate of development of lesions. MRI performed during on-treatment period provided data for comparison with pretreatment period. MRI examinations of the brain were standardized to ensure sufficient image quality for the evaluation of radiographic progression of multiple sclerosis. This assessment was performed in the NMR Unit of The Institute of Neurology, UCL, Queen Square. Outcome assessments for biomarkers were collected at visits 1-8 and transferred to the Blizard Institute laboratory for analysis. The Institute for Biomedicine at the Aarhus University in Denmark were also contracted to analyse virology samples for HERVs according previously published methodology (22). In summary, The expression of HERV encoded proteins was assessed by flow cytometric analysis on isolated PBMCs using a Novocyte Acea Bioscences Flow cytometer. Polyclonal antibodies against HERV peptide antigens were raised in New Zealand White rabbits. The peptide epitopes are localized at equivalent positions in ORFs at the respective HERV loci. Both peptides and antisera were by Sigma Genosys (UK). Antibody binding to targets was visualised using goat anti-rabbit Alexa 488. The peptide antigens were: HERV-W/MSRV Env W1 and W3 (aa415-431, and aa301-317, respectively, AF208161.1), HERV-W/MSRV Gag (aa 231-246, AF123881.1); HERV-H/F Env H1 and H3 (aa489-505, and aa 370-386, respectively, AJ289709), and HERV-H/F Gag (aa380-395, AL354685). Preimmune sera were collected from all rabbits before immunization, and fluorescence indexes (Flix) determined as fluorescence intensity of immune vs preimmune serum. Staining for B cells and monocytes, respectively, was performed using mouse anti-human anti-CD3 eFluor 450, mouse anti-human anti-CD14 PE, and mouse antihuman anti-CD20 APC.

Data collected included gender, age, age of onset, ethnicity. Assessments conducted during the visits included neurological examination and EDSS. Blood and saliva samples were collected for biochemical, virological and viral immunological analysis including exposure to other viruses (HSV, VZV, EBV, CMV, and HHV-6) and inflammatory markers. The following quality of life parameters were measured at each trial visit: Patient Questionnaire using the SF36 - Physical Functional Score; Role-Physical Scale ; Bodily Pain Scale; General Health Scale ; Vitality Scale ; Social Functioning Scale ; Role Emotional Scale; Mental Health Scale. Patient Questionnaire using the Modified Fatigue Assessment Scale and the MOS Pain Effects Scale Assessment.

\section{Statisticalmethods}

The primary efficacy analysis was a comparison between the rate of $\mathrm{Gd}$ enhancing T1-weighted lesions appearing on the monthly brain MRI over the treatment period, compared to the pretreatment observation period. The sample size was based on Sormani et al(23), indicating that for a before-after design with number of Gd-enhancing lesions counted at baseline, 19 patients are required to detect, with $80 \%$ power at $5 \%$ significance, a $40 \%$ reduction in the number of $\mathrm{Gd}$ enhanced lesions. We planned to enrol 24 to allows for patient dropout. The analysis was performed using the counts of new or persisting lesions as the outcome variable in a mixed effects Poisson regression, with a linear predictor containing a binary indicator for the counts occurring on 
treatment vs pre-treatment, and as covariate the baseline (visit 2) count for each patient (log transformed for consistency with the Poisson model). This type of model, which allows for withinpatient lack of independence, estimates the count rates before and on treatment, and the corresponding rate ratio with a Wald test of this ratio. A second mixed effects Poisson regression with the same outcome used a linear predictor containing the month of follow-up and an interaction between month and the binary indicator above, with the baseline visit count as covariate. This model estimates the change in monthly rates over time, in other words, the count 'gradient' during each period, and tests (Wald) for a change in gradient: it is important to estimate these gradients because if there were a decline already during the pre-treatment period, a lower overall treatment lesion rate could not plausibly be attributed to the treatment, since it could reflect a decline over time independent of, and starting before, treatment. T2-weighted lesions counts, which were only observed twice, at visits 5 (before) and 8 (after treatment), were compared with a Wilcoxon signedranks test of the within-person changes. Linear mixed models were used similarly to compare mean values of continuous outcomes before and during treatment (with similar linear predictors to Poisson models, and again with additional models estimating gradients before vs during treatment), of a) specific inflammatory markers of MS; b) specific indicators of endogenous retroviral and herpes virus activity; c) clinical and quality of life measures. Linear mixed models with HERV outcomes and time-varying lesion counts as predictors were used to investigate potential associations between these two measures at any given time-point.

Results

A total of 31 participants were screened; of these 8 had no evidence of $\mathrm{Gd}$ enhancing lesions in their baseline MRI and were not eligible to enter the baseline phase. Of the 23 participants who were recruited into the study 3 were withdrawn prior to starting the treatment phase; one at the request of the participant and the remaining two due to MS relapse. 20 participants entered the treatment phase and completed the 12 week treatment period. They received the recommended dose of open label Isentress-Raltegravir (MA number: EU/1/07/436/001) 400mg twice a day administered as a potassium salt in a film coated tablet.

The intention-to-treat (ITT) population includes all participants recruited who received at least one dose of study medication. Data for all 20 participants enrolled in the study was used in the ITT analysis. There were four participants who were regarded as being protocol deviations; two due to an error in the original protocol and two due to intermittent use of inhaled steroids for a respiratory illness that was temporary. The Investigators determined the reasons for these deviations did not influence the potential outcomes of the trial and as all participants finished the 24 weeks of baseline and treatment, data are presented as an ITT analysis. Participant characteristics are given in Table 1, showing a majority of females, minimal disability, but quite active RRMS in terms of relapses.

Table 1: Baseline Characteristics

\begin{tabular}{|l|l|}
\hline \multicolumn{1}{|c|}{ Characteristic } & \\
\hline Age, years: mean (SD) & $41(7.0)$ \\
\hline Female: $\mathrm{n}(\%)$ & $14(70 \%)$ \\
\hline $\begin{array}{l}\text { Number of relapses in } \\
\text { past year: mean (SD) }\end{array}$ & $1.50(0.61)$ \\
\hline $\begin{array}{l}\text { Baseline EDSS: } \\
\text { mean, median (SD) }\end{array}$ & $2.40,2.50(1.03)$ \\
\hline
\end{tabular}


There were no clinically significant biochemical safety issues requiring either participant withdrawal or variation of the prescribed dose and/or frequency of raltegravir administration. The mean number of visits with any adverse events were very similar during the baseline phase (2.35) as during the treatment phase (2.30); there were a total of 245 adverse events in the 31 screened patients with one SAE due to a hospitalisation, for social reasons, which was considered not to be associated with raltegravir.

\section{Treatment Effect \\ Gd Enhanced MRI outcomes}

T1 lesions (i.e. Gd-enhancing)

No statistically significant differences were found in the after vs before medication periods for either $\mathrm{T} 1$ or $\mathrm{T} 2$ weighted lesion counts, or for the T1 lesions broken down by new or persisting lesions. The mean number of T1 lesions per visit was 3.08 (range 0-16) and the median number was 2 . Visit 2 was the enrolment visit when all participants needed to have at least one Gd-enhanced lesion in order to enter the baseline phase. This visit is excluded from the comparative before versus after analysis.

Table 2 compares the counts and rates for new and persisting T1 Gd-enhanced lesions, showing a very slight and non-significant increase in rate for the on-treatment ys pre-treatment period (rate ratio $1.04, \mathrm{p}=0.681$ ).

Table 2: Counts and rates for new plus persisting T1 Gd-enhanced lesions

\begin{tabular}{|c|c|c|c|c|c|c|}
\hline & \multicolumn{2}{|c|}{$\begin{array}{c}\text { Before treatment } \\
\text { (visits } 3,4,5 \text { ) }\end{array}$} & \multicolumn{2}{|c|}{$\begin{array}{l}\text { On treatment } \\
\text { (visits } 6,7,8 \text { ) }\end{array}$} & \multirow[b]{2}{*}{$\begin{array}{l}\text { Rate } \\
\text { ratio }^{a}\end{array}$} & \multirow{2}{*}{$\begin{array}{l}95 \% \text { Confidence } \\
\text { interval; p-value }\end{array}$} \\
\hline & $\begin{array}{l}\text { Total } \\
\text { count }\end{array}$ & $\begin{array}{l}\text { Monthly } \\
\text { rate }\end{array}$ & $\begin{array}{l}\text { Total } \\
\text { count }\end{array}$ & $\begin{array}{c}\text { Monthly } \\
\text { rate }\end{array}$ & & \\
\hline Mean & 8.65 & 2.89 & 9.05 & 3.02 & 1.04 & $0.85,1.29 ; p=0.681$ \\
\hline Median & 6.00 & 2.00 & 6.00 & 2.00 & & \\
\hline Min & 0 & 0.00 & & 0.00 & & \\
\hline Max & 32 & 10.67 & 31 & 10.33 & & \\
\hline
\end{tabular}

The difference in the rates of change ('gradients') of the monthly counts before vs on treatment was also non-significant, $P=0.659$.

Separate comparisons of new and persisting $\mathrm{T} 1 \mathrm{Gd}$-enhanced lesions gave rate ratios, on vs pretreatment, which showed a small non-significant increase for new (rate ratio 1.16, $p=0.314$ ) and a small non-significant decrease (rate ratio $0.93, p=0.652$ ) for persisting lesions.

\section{T2 Weighted lesions}

Table 3 compares total counts of new or enlarging T2-weighted lesions at visit 5 (before) and visit 8 (after treatment) and shows a very slight and non-significant increase in these lesions.

\section{Table 3. T2-weighted new or enlarging lesions before and after treatment}

$\begin{array}{ll}\begin{array}{l}\text { Before treatment } \\ \text { (visit 5) count }\end{array} & \begin{array}{l}\text { After treatment } \\ \text { (visit 8) count }\end{array} \\ \text { Count ratio } & \text { p-value }\end{array}$




\begin{tabular}{l|r|r|r|c|}
\cline { 2 - 5 } Mean & 4.45 & 4.65 & 1.05 & 0.472 \\
\cline { 2 - 5 } Median & 3.00 & 3.00 & & - \\
\cline { 2 - 5 } $\operatorname{Min}$ & 0 & 0 & & - \\
\cline { 2 - 5 } & 17 & 20 & & - \\
\cline { 2 - 4 } & ${ }^{a}$ Wilcoxon signed-ranks test of within-person changes & \\
\cline { 2 - 4 }
\end{tabular}

Virologic Results

Human endogenous retrovirus(HERV)

Quantitative measurement of HERVs were conducted in the Department of Biomedicine, University of Aarhus, Denmark by Tove Christensen. The HERVs were HERV-W/MSRV); and HERVH/F. There was no significant association between the quantitation of either HERV and baseline or treatment phases.

While there was no evidence of association with treatment, there was an independent significant association between the number of new $\mathrm{T} 1 \mathrm{Gd}$-enhancing lesions and an increase in expression of HERV-W Gag on B cells; while overall HERV-W/MSRV Gag Flix B cells values varied during both trial periods, throughout the trial higher T1 Gd-enhanced lesion counts at any individual visit were associated with higher values of HERV-W/MSRV Gag Flix B cells values at that visit: entering the monthly Gd lesion counts into the linear mixed model for ITT suggests that during the trial period, having one additional lesion at a visit predicts a HERV-W/MSRV Gag Flix B cells increase of 0.008 (dimensionless, $95 \% \mathrm{Cl} .00082, .01521$ ), $\mathrm{P}=0.029$. This was also reflected in a borderline significant association whereby patients with larger decreases in mean HERV-W/MSRV Gag Flix B cells values during the two trial periods tended to have larger decreases in corresponding mean T1 Gd-enhanced mean lesion count: Spearman $r=0.46, P=0.057$.

Epstein - Barr virus (EBV)

There were no associations between quantitative PCR measurements of EBV in saliva specimens and number of $\mathrm{T} 1$ lesions. When EBV was dichotomized $(0 />0)$, there was a lower odds of having EBV $>0$ with a greater number of total lesions (odds ratio 0.79 per lesion, $P=0.081$ ); this was more probably driven by new lesions $(P=0.097)$ than persisting lesions $(P=0.442)$. However, the number of analyzable saliva samples was relatively small. It was observed that about $40 \%$ of participants shed $E B V$ at some time during the six months of saliva collection and that the cross-sectional prevalence of EBV shedding was about $15 \%$.

\section{Inflammatory Markers}

No change was detected in inflammatory markers (IL-8, IL-1ß, IL-6, IL-10, TNF, IL-12p70 and HCRP), which were all within normal limits during the baseline phase and during the treatment phase. Serum CD163 expression was also unchanged by Raltegravir.

\section{Disability Measurements}


There was no statistically significant change in either the means or gradients for EDSS and the 25FTW. There was a significant improvement in both the PASAT and the 9HPTmean scores during the course of the trial, but the improvement gradient was similar in both trial periods, suggesting either a learning effect or a trial participation effect.

Quality-of-life Measurements

Table 5: Quality of Life Scores SF36; MOS Fatigue Assessment and PES

\begin{tabular}{|c|c|c|c|}
\hline Measure & $\mathbf{n}$ & Change in means & P-value ${ }^{c}$ \\
\hline PQ - SF36 - Physical Functional Score ${ }^{a}$ & 20 & -0.92 & 0.685 \\
\hline PQ - SF36 - Role-Physical Scale ${ }^{a}$ & 20 & -9.48 & 0.034 \\
\hline PQ - SF36 - Bodily Pain Scale ${ }^{a}$ & 20 & -5.42 & 0.02 \\
\hline PQ - SF36 - General Health Scale ${ }^{a}$ & 20 & -4.67 & 0.00 \\
\hline PQ - SF36 - Vitality Scale ${ }^{a}$ & 20 & 5.06 & \\
\hline$P Q$ - SF36 - Social Functioning Scale ${ }^{a}$ & 20 & 2.45 & 0.4 \\
\hline PQ - SF36 - Role Emotional Scale ${ }^{a}$ & 20 & -8.62 & 0.0 \\
\hline PQ - SF36 - Mental Health Scale ${ }^{a}$ & 20 & 5.72 & 0.013 \\
\hline$P Q$ - PFA - Patient Fatigue Assessment ${ }^{b}$ & 20 & -1.51 & 0.683 \\
\hline$P Q$ - PPA - Patient Pain Assessment ${ }^{b}$ & 19 & 4.07 & 0.14 \\
\hline PQ - MSIS - Total Score ${ }^{b}$ & 20 & -2.10 & 0.220 \\
\hline PQ - MSWS-12 - MSWS Total Score ${ }^{b}$ & 20 & -0.35 & 0.772 \\
\hline
\end{tabular}

There appeared to be significant changes in some of the Quality of Life parameters during the course of the trial, but these changes are not plausibly attributable to treatment, as mentioned later.

\section{Discussion}

This Phase 2 baseline versus treatment open label clinical trial was designed to determine the efficacy of the integrase strand transfer inhibitor, raltegravir, in patients with active relapsing remitting multiple sclerosis. The aim of the trial was to investigate if raltegravir may inhibit a HERV and thereby ameliorate the possible role of HERV in active RRMS as measured by Gd-enhanced lesion development. Raltegravir did not provide statistically significant evidence of efficacy in the primary outcome measure, reduction in the number and rate of development of T1 lesions by Gdenhanced MRI during the treatment phase compared with the baseline phase. An alternative outcome measure to enhancing lesions seen on monthly Gd-enhanced MRI is the number of new or enlarging T2 lesions seen on T2-weighted scans over longer intervals. In our study, this outcome measure was analysed for the 3-month baseline and treatment periods and showed no evidence for treatment efficacy (Table 3). There was also no improvement in the secondary outcomes of quantitative virological measurements or inflammatory markers. Improvements in disability or quality-of-life measures are most probably attributable to a placebo effect of treatment or of general trial participation. Raltegravir did not produce any significant increase in adverse events during the treatment phase compared with the non-treatment baseline phase. Two experimental findings, unrelated to treatment, were interesting and previously unreported: that HERV-W tended to increase in association with increasing number of new T1 lesions and quantitative EBV shedding in saliva also paralleled increase in observed new T1 lesions. However, these associations must be 
interpreted with caution and regarded only as hypothesis generating for future confirmation, since they are vulnerable to type I statistical error.

There are a number of possible reasons why raltegravir appears to have had no observed efficacy in this clinical trial. The clinical trial design, which was taken from the model proposed by Somani et al (23). was considered the most feasible in the circumstances of not having extensive evidence of effect in animal models. The trial was of limited duration with the baseline and treatment phases each of 12 weeks which was determined by the investigators as a clinically reasonable period for which patients with active disease could remain not taking a disease modifying therapy. The single agent, raltegravir, was not in a combination of antiretroviral therapies as has been shown to be effective in treating the exogenous retrovirus, HIV. While there is no direct link between HIV therapy and the possibility that MS is triggered or caused by a HERV, we believe it would have been preferable to administer a combination of antiretroviral agents that act on different phases in the possible life-cycle of an endogenous retrovirus. This idea may be supported by the NHS record linkage study that found a significantly reduced incidence of MS in patients who had HIV infection compared with a large control population. The possibility that these patients were also taking combination ARVs supports the hypothesis that ARVs may influence the activity of a HERV which could trigger or cause MS (24).

Given that patients received just 12 weeks of raltegravir administration, the ARV would need to have had an impact almost immediately in order to cause a biological (anti-inflammatory) effect on the development of new Gd-enhancing lesions. We know this is not the case with HIV infection where it may take some weeks for an ARV combination to fully suppress HIV. While there are no in-vivo data on HERVs, it is possible that it may require weeks or months of treatment to achieve a therapeutic effect on an endogenous retrovirus. The dose and frequency of raltegravir was chosen as this is the licensed regimen for treatment of HIV infection. However, there are no data on the use of raltegravir in RRMS and treating possible HERV production. It is possible the dose level and frequency may need to be much higher than for HIV; it is possible that the dose and frequency of administration should be different in RRMS. This observation has been recently supported by laboratory data for HERV-K, which although not a purported caùse of MS, provides some indication as to the required concentration of raltegravir to inhibit a HERV. The linkage between HERVs and MS is being explored in a Phase $2 \mathrm{~b}$ clinical trial that is investigating the use of GNbAC1 at three dose levels compared with placebo in 270 patients who have RRMS (25). This trial has fully enrolled and the results should provide information on the role of a humanized monoclonal antibody against the envelope protein of HERV-K (MSRV). This trial should further advance the field of investigating the possible role of HERVs and MS.

In conclusion, the INSPIRE trial was the first clinical study on using an HIV antiretroviral therapy to treat RRMS. While the results did not demonstrate a statistically significant improvement in either the primary or secondary outcomes, it did provide another step in the search for an aetiology of multiple sclerosis. 
1. Charcot JM. Histologie de la sclerose en plaques Gaz Hop (Paris) 1868: 41: 554-5, 557-8, 566

2. Compston $\mathrm{A}$. The $150^{\text {th }}$ anniversary of the first depiction of the lesions of multiple sclerosis $\mathrm{J}$ Neurol Neurosurg Psychiatry 1988; 51: 1249-1252

3. Gourrand PA Harbo H.F. Hauser. S.E. et al The genetics of Multiple sclerosis: an up-to-date review. Immunol Rev 2012; 248: 87-103

4. Goodin D.S. The causal cascade to multiple sclerosis: a model for MS pathogenesis PLoS ONE 4 2009; e4565. (2) Feb 26

5. Kempkes B, Robertson E. S. Epstein-Barr Virus latency: current and future perspectives. Current Opinion in Virology 2015; $14: 138-144$

6. Baker D, Marta M, Giovannoni G, Schmierer K. Memory B Cells are Major Targets for Effective Immunotherapy in Relapsing Multiple Sclerosis. EBioMedicine. 2017 Feb;16:41-50

7. Chastain EM, Miller SD. Molecular mimicry as an inducing trigger for CNS autoimmune demyelinating disease. Immunol Rev. 2012 Jan; 245(1):227-38.

8. Balada E, Ordi-Ros J, Vilardell-Torres M: Molecular mechanisms mediated by human endogenous retroviruses (HERVs) in autoimmunity Rev Med Virol, 2009; 19 :273-86

9. Punkosdy GA, Blain M Glass DD et al. T-cell expansion during chronic viral infection is dependent on endogenous retroviral superantigens. Proc Natl Acad Sci U S A. 2011 Mar 1;108(9):3677-82

10. Hansen DT, Petersen T, Christensen T. Retroviral envelope proteins: Involvement in neuropathogenesis Journal of the Neurological Sciences 2017, 380: 151-163

11. Perron $\mathrm{H}$, Lolande B, Gratacap B, Laurent $\mathrm{O}$ et al. Isolation of retroviruses from patients with multiple sclerosis. Lancet 1991; 337: 862-863

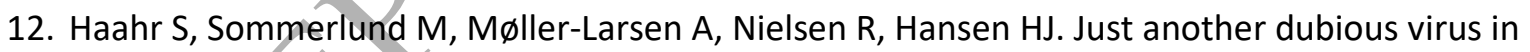
cells from a patient with multiple sclerosis? Lancet. 1991 Apr 6;337(8745):863-4

13. Christensen T, Dissing Sørensen $P$, Riemann $H$, Hansen HJ, Møller-Larsen A. Expression of sequence variants of endogenous retrovirus RGH in particle form in multiple sclerosis. Lancet. 1998 Sep 26;352(9133):1033

14. Christensen T. Association of human endogenous retroviruses with multiple sclerosis and possible interactions with herpes viruses Rev Med Virol, 2005; 15(3) 179-211

15. Morandi E Tanasescu R, Tarlinton RE et al The association between human endogenous retroviruses and Multiple Sclerosis: A systematic review and meta-analysis. PLoS ONE 2017. 12(2): e0172415

16. Grandi N, Cadeddu M, Blomberg J, Tramontano E. Contribution of type W human endogenous retroviruses to the human genome: characterization of HERV-W proviral insertions and 
processed pseudogenes. Retrovirology. 2016;13(1):67

17. Brudek T, Christensen T, Aagaard L, Petersen T, Hansen HJ, Møller-Larsen A. B cells and monocytes from patients with active multiple sclerosis exhibit increased surface expression of both HERV-H Env and HERV-W Env, accompanied by increased seroreactivity. Retrovirology. 2009;6: 104

18. Mameli G, Poddighe L, Mei A, Uleri E, Sotgiu S, Serra C, Manetti R, Dolei A. Expression and activation by Epstein Barr virus of human endogenous retroviruses-W in blood cells and astrocytes: inference for multiple sclerosis. PLoS One, 2012. 7(9): p. e44991.

19. Berger JR, Sheremata WA, Resnick L et al. Multiple sclerosis -like illness occurring with human immunodeficiency virus infection Neurology 1989 39(3): 324-9

20. Maruszak H, Brew BJ, Giovannoni G, Gold J. Could antiretroviral drugs be effective in multiple sclerosis? A case report Eur J Neurol 2011 18(9) e110-1

21. Liedthe MD, Ryan Tomlin C, Lockhart SM et al. Longterm efficacy and safety of raltegravir in the management of HIV infection Infect Drug Resist, 2014; $7: 73-84$

22. Møller-Larsen A, Brudek T, Petersen T, Petersen EL, Aagaard M, Hansen DT, Christensen T. Flow cytometric assay detecting cytotoxicity against human endogenous retrovirus antigens expressed on cultured multiple sclerosis cells. Clin Exp Immunol. 2013 Sep;173(3):398-410)

23. Sormani MP, Miller DH, Couli G et al. Clinical trials of multiple sclerosis monitored with enhanced MRI; new sample size calculations based on large data sets.J Neurol, Neurosurg Psychiatry 2001; $70: 494-499$

24. Gold J., Goldacre R., Maruszak H., et al. HIV and lower risk of multiple sclerosis: beginning to unravel a mystery using a record-linked database study J Neurol Neurosurg Psychiatry 201586 (1): 9-12.

25. Kremer D., Glanzman R., Traboulsee A., et al. Prehistoric enemies within: The contribution of human endogenous retroviruses to neurological diseases. Meeting report: "Second International Workshop on Human Endogenous Retroviruses and Human Disease". Washington DC, March 13 and 14, 2017. Multiple Sclerosis and Related Disorders 2017; 15: 1823

Disclosure: We certify that no party involved in this study has a direct financial interest in the results of the research supporting this article has or will confer a benefit on us or on any organization with which we are associated.

Acknowledgements: This study was funded by Merck, Sharpe and Dohme 